\title{
Effect of $n-3$ PUFA supplementation at different EPA:DHA ratios on the spontaneously hypertensive obese rat model of the metabolic syndrome
}

\author{
Eunice Molinar-Toribio ${ }^{1}$, Jara Pérez-Jiménez ${ }^{1 *}+$, Sara Ramos-Romero ${ }^{1,2}$, Marta Romeu ${ }^{3}$, \\ Montserrat Giralt ${ }^{3}$, Núria Taltavull ${ }^{3}$, Mònica Muñoz-Cortes ${ }^{3}$, Olga Jáuregui ${ }^{4}$, Lucía Méndez ${ }^{5}$, \\ Isabel Medina ${ }^{5}$ and Josep Lluís Torres ${ }^{1}$ \\ ${ }^{1}$ Institute of Advanced Chemistry of Catalonia (IQAC-CSIC), Barcelona, Spain \\ ${ }^{2}$ Biomedical Research Networking Center in Bioengineering, Biomaterials, and Nanomedicine (CIBER-BBN), \\ Zaragoza, Spain \\ ${ }^{3}$ Unidad de Farmacología, Facultad de Medicina y Ciencias de la Salud, Universidad Rovira $i$ Virgili, Reus, Spain \\ ${ }^{4}$ Scientific and Technological Centers of the University of Barcelona (CCiT-UB), Barcelona, Spain \\ ${ }^{5}$ Instituto de Investigaciones Marinas (IIM-CSIC), Vigo, Spain \\ (Submitted 17 June 2014 - Final revision received 3 December 2014 - Accepted 17 December 2014 - First published online 27 February 2015)
}

\begin{abstract}
The increasing incidence of the metabolic syndrome (MetS), a combination of risk factors before the onset of CVD and type 2 diabetes, encourages studies on the role of functional food components such as long-chain $n-3$ PUFA as preventive agents. In the present study, we explore the effect of EPA and DHA supplementation in different proportions on spontaneously hypertensive obese (SHROB) rats, a model for the MetS in a prediabetic state with mild oxidative stress. SHROB rats were randomised into four groups $(n 7)$, each supplemented with EPA/DHA at ratios of 1:1, 2:1 and 1:2, or soyabean oil as the control for 13 weeks. The results showed that in all the proportions tested, EPA/DHA supplementation significantly lowered total and LDL-cholesterol concentrations, compared with those of the control group. EPA/DHA supplementation at the ratios of 1:1 and 2:1 significantly decreased inflammation (C-reactive protein levels) and lowered oxidative stress (decreased excretion of urinary isoprostanes), mainly at the ratio of 1:2. The activity of antioxidant enzymes increased in erythrocytes, abdominal fat and kidneys, with magnitudes depending on the EPA:DHA ratio. PUFA mixtures from fish affected different MetS markers of CVD risk factors in SHROB rats, depending on the ratios of EPA/DHA supplementation. The activation of endogenous defence systems may be related to the reduction of inflammation and oxidative stress.
\end{abstract}

Key words: Metabolic syndrome: $n$-3 PUFA: Spontaneously hypertensive obese rats: EPA/DHA

The metabolic syndrome (MetS) is a combination of features such as insulin resistance hypercholesterolaemia, hypertriacylglycerolaemia, hypertension or obesity ${ }^{(1)}$ that makes subjects with the MetS highly prone to developing type 2 diabetes mellitus and CVD. The MetS is also accompanied by low-grade chronic inflammation $^{(2)}$ and increased oxidative stress (OS) ${ }^{(3)}$.

The increasing incidence of the MetS has encouraged the search for preventive strategies, based on either drugs or dietary approaches, including diet supplementation with bioactive compounds from food sources. Long-chain $n-3$ PUFA of marine origin are key components of cell membranes that may be used as a functional food and dietary supplement.
The two most common $n$-3 PUFA in fish oils are EPA $(20: 5)$ and DHA (22:6). Studies using different animal models, such as rats with sucrose-induced or fructose-induced MetS, have shown that regular supplementation with n-3 PUFA decreases blood pressure and levels of insulin, TAG, cholesterol, NEFA or total lipids ${ }^{(4,5)}$. Because $n-3$ PUFA play the role of mediators in nuclear events, they modulate these parameters via different mechanisms, including interactions with other nutrients and effects on the expression of genes involved in lipid and glucose metabolism ${ }^{(6)}$.

In human subjects, $n-3$ PUFA have been shown to modulate certain characteristic MetS features such as insulin resistance,

Abbreviations: CAT, catalase; CRP, C-reactive protein; GPx, glutathione peroxidase; GR, glutathione reductase; GSH, reduced glutathione; GSSG, oxidised glutathione; IsoP, isoprostanes; LC-ESI-QqQ-MS/MS, liquid chromatography coupled to tandem MS after electrospray ionisation; LDL-C, LDL-cholesterol; LDL-ox, oxidised LDL; MetS, metabolic syndrome; OS, oxidative stress; SHROB, spontaneously hypertensive obese; SOD, superoxide dismutase; WKY, Wistar Kyoto.

* Corresponding author: J. Pérez-Jiménez, email jara.perez@ictan.csic.es

†Present address: Department of Metabolism and Nutrition, Institute of Food Science, Technology and Nutrition (ICTAN-CSIC), Madrid, Spain 
hypertension and dyslipidaemia, although their effects on other aspects of CVD, such as the incidence of myocardial infarction or arrhythmia, remain controversial ${ }^{(7)}$. However, it should be noted that, to date, most trials have not considered that the relative proportion of EPA and DHA may influence the results, which might explain some of these controversies. Indeed, it has been observed that different proportions of $n-3$ and $n-6$ PUFA have different effects on health ${ }^{(8)}$, so different proportions within the $n-3$ series may also differentially affect MetS risk factors. Recently, we compared the effects of supplementation with EPA/DHA at different ratios on the markers of CVD and OS in healthy Wistar Kyoto (WKY) rats, and found that the proportion 1:1 was especially favourable for the improvement of some risk factors of type 2 diabetes mellitus, such as glycated $\mathrm{Hb}$ levels, as well as for a reduction in OS, as shown by increased plasma antioxidant capacity and decreased protein oxidation ${ }^{(9,10)}$.

Spontaneously hypertensive obese (SHROB or Koletsky) rats are an animal model of the MetS. The strain exhibits a mutation that introduces a premature stop codon in the extracellular leptin receptor domain, which results in truncated receptors that are insensitive to leptin ${ }^{(11,12)}$. The rats are obese, hypertensive and hyperinsulinaemic, although they are not hyperglycaemic. They have been suggested as an appropriate model for the MetS in a prediabetic state $^{(13,14)}$. The aim of the present study was to evaluate the effect of supplementation with EPA/DHA mixtures at different proportions found in fish and fish oil-based supplements, namely $1: 1,2: 1$ and $1: 2$, on the markers of CVD and OS in SHROB rats, as an animal model for the MetS. To the best of our knowledge, the present study was the first to investigate the effect of $n-3$ PUFA in SHROB rats.

\section{Materials and methods}

\section{Materials and reagents}

The standard A04 diet was obtained from Harlan Iberica. Oils with different EPA:DHA ratios were obtained by mixing appropriate amounts of the commercial fish oils AFAMPES $121 \mathrm{EPA}$ (AFAMSA), EnerZona Omega $3 \mathrm{RX}$ and Oligen liquid DHA $80 \%$ (IFIGEN-Equip 98, S.L.). Soyabean oil, obtained from unrefined organic soyabeans (first cold pressing), was from Clearspring Limited. Other reagents and chemicals were as follows: ketamine chlorhydrate from Merial Laboratorios; xylacine from Quimica Farmaceutica; $\beta$-glucuronidase $(90 \mathrm{U} / \mu \mathrm{l})$ from Sigma-Aldrich. Also, $15-\mathrm{F}_{2 \mathrm{t}}$-isoprostanes (IsoP), $\left[{ }^{2} \mathrm{H}_{4}\right] 15-$ $\mathrm{F}_{2 \mathrm{t}}$-IsoP, 5- $\mathrm{F}_{2 \mathrm{t}}$-IsoP, $5-\mathrm{F}_{2 \mathrm{c}}$-IsoP, 8- $\mathrm{F}_{2 \mathrm{t}}$-IsoP, 15- $\mathrm{F}_{3 \mathrm{t}}$-IsoP and 2,3-dinor-15- $\mathrm{F}_{2 \mathrm{t}}$-IsoP were obtained from Cayman Chemical, which were used without further purification.

Ethanol (analytical grade) was purchased from Carlo Erba. Methanol, heptane, acetonitrile (all HPLC grade) and formic acid (analytical grade), hydrochloric acid fuming 37\%, ethanol (absolute), ethyl acetate, chloroform, $\mathrm{Na}_{2} \mathrm{CO}_{3}, \mathrm{NaHCO}_{3}$, EDTA, $O$-phthalaldehyde, $N$-ethylmaleimide and albumin were obtained from Merck. Iodoacetamide, EDTA, Tris- $\mathrm{HCl}$, acetone, phosphomolybdic acid, Bradford reagent, NADPH, GR (glutathione reductase from baker's yeast), GSH (L-glutathione reduced, minimum 99\%), tert-butyl hydroperoxide solution, GSSG (L-glutathione oxidised) and fluorescein-5-thiosemicarbazide were all obtained from Sigma-Aldrich. N,O-Bis(trimethylsilyl)trifluoro-acetamide was from Supelco. Drabkin's reagent was purchased from Química Clínica Aplicada. $\mathrm{Na}_{2} \mathrm{HPO}_{4}$, $\mathrm{NaH}_{2} \mathrm{PO}_{4}, \mathrm{NaCl}$, epinephrine, $\mathrm{KH}_{2} \mathrm{PO}_{4}, \mathrm{~K}_{2} \mathrm{HPO}_{4}$, TCA and $\mathrm{NaOH}$ were purchased from Panreac Química. Water for the assay solutions was obtained using a Milli-Q water purification system from Millipore Corporation.

\section{Animals}

A total of twenty-eight female SHROB rats (Charles River Laboratories), aged 11-14-weeks, were used for the present study. They were kept in a room containing no male animals to minimise the influence of hormonal alterations. All the procedures strictly adhered to the European Union guidelines for the care and management of laboratory animals, and approved by the CSIC Subcommittee of Bioethical Issues (reference no. AGL2009-12 374-C03-03).

\section{Experimental design}

Rats were kept in Makrolon cages ( $n$ 2-3 per cage) under controlled conditions of stable humidity $(50 \pm 10 \%)$ and temperature $\left(22 \pm 2^{\circ} \mathrm{C}\right)$ with a $12 \mathrm{~h}$ light $-12 \mathrm{~h}$ dark cycle. Rats were randomly divided into four groups, each $(n 7)$ supplemented with a different oil mixture: EPA/DHA 1:1, EPA/DHA 2:1, EPA/DHA 1:2 or soyabean oil as the control (isoenergetic without $n$-3 long-chain PUFA). After 1 week of acclimatisation, all the groups were fed a standard pelleted A04 diet, given water (Ribes) ad libitum, and orally administered with the corresponding oil mixture or soyabean oil at a weekly dose of $0.8 \mathrm{ml} / \mathrm{kg}$ body weight for 13 weeks. All the diets had similar fat and energy content (see online supplementary Table S1). Moreover, the weekly dose of oil orally administered did not alter the overall intake of the animals in terms of macronutrients, compared with a standard diet, with $20 \%$ of energy derived from proteins, $67 \%$ from carbohydrates and 13\% from fat (see online supplementary Table S1). The fatty acid composition of the supplements is also provided in Table S2 (available online). Because PUFA are extremely susceptible to oxidation and the byproducts are potentially toxic, lipid oxidation levels were checked throughout the experiment (peroxide values $<5 \mathrm{mEq}$ oxygen/kg oil). Intragastric administration was selected because PUFA have a strong tendency to oxidise when incorporated into the animal feed, as demonstrated by our previous study (data not shown). Oxidised PUFA may seriously alter the results, as described for commercial pills ${ }^{(15)}$. After week 16, rats were fasted overnight, anaesthetised intraperitoneally with ketamine and xylacine (80 and $10 \mathrm{mg} / \mathrm{kg}$ body weight, respectively), and then killed by exsanguination.

\section{Sample collection}

For urine collection, at week 14/15 of the experiment, the rats were placed in metabolic cages and deprived of food for $18 \mathrm{~h}$, 
after which time the samples were collected. After 1 week, blood was collected by cardiac puncture under anaesthesia, and plasma, serum and erythrocytes were also collected. Abdominal fat was weighed and immediately frozen. Tissue samples collected from the heart, brain, liver and kidneys were washed with $0.9 \% \mathrm{NaCl}$ solution, dried, weighed and immediately frozen in liquid $\mathrm{N}_{2}$. All the samples were stored at $-80^{\circ} \mathrm{C}$ until analysis. Before analysis, the tissue samples were homogenised with sodium phosphate buffer and ultra-centrifuged.

\section{CVD risk factors}

Physical measurements. Body weight was monitored weekly throughout the experiment. Abdominal fat was collected post-mortem, weighed and expressed as a percentage of body weight.

Determination of plasma lipid profile. Plasma total cholesterol, LDL-cholesterol (LDL-C), HDL-cholesterol and TAG, as well as ApoA1 and ApoB100 concentrations were determined by spectrophotometric methods using the corresponding kits from Cusabio Biotech Co. Plasma oxidised LDL (LDL-ox) was determined using an ELISA kit (Cusabio Biotech Co.) by measuring absorbance at $450 \mathrm{~nm}$ with a PowerWave XS2 spectrophotometer (Biotek Instruments, Inc.).

Determination of glycaemia. Blood glucose levels were determined by the enzyme electrode method using an Ascensia ELITE XL blood glucose meter (Bayer Consumer Care). Plasma insulin level was determined using an ELISA kit (Millipore). Blood glycated $\mathrm{Hb}$ level was measured using a spectrophotometric kit (Spinreact).

Markers of endothelial function, inflammation and thrombotic activity. The corresponding ELISA kits from Cusabio Biotech Co. were used to measure the following parameters in plasma: vascular cell adhesion molecule-1 and intercellular adhesion molecule-1, as markers of endothelial function; C-reactive protein (CRP, detection range $0 \cdot 16-10 \mathrm{ng} / \mathrm{ml}$ ), as an inflammation marker; plasminogen activator inhibitor-1, as a marker of thrombotic activity.

\section{Endogenous antioxidant systems}

The activities of superoxide dismutase (SOD) and catalase (CAT) were measured in erythrocytes, kidneys, abdominal fat, heart and brain using standard spectrophotometric methods $^{(16,17)}$.

GR and glutathione peroxidase (GPx) activities were determined in erythrocytes and kidneys by spectrophotometric methods ${ }^{(18,19)}$. GSH and GSSG activities were determined in kidneys using fluorometric methods at wavelengths of $350 \mathrm{~nm}$ (excitation) and $420 \mathrm{~nm}$ (emission) ${ }^{(20)}$. Measurements in erythrocytes were normalised to the amount of $\mathrm{Hb}$ by the Drabkin method $^{(21)}$, while those in tissues were normalised to the amount of protein estimated by the Bradford method ${ }^{(22)}$.

\section{Isoprostanes}

IsoP of the $\mathrm{F}_{2}$ and $\mathrm{F}_{3}$ series were determined in urine samples by liquid chromatography coupled to tandem MS after electrospray ionisation (LC-ESI-QqQ-MS/MS), according to a procedure described previously ${ }^{(23,24)}$, with some modifications. Briefly, stock standard solutions of all IsoP were prepared in ethanol $(1 \mathrm{ng} / \mu \mathrm{l})$ and stored at $-20^{\circ} \mathrm{C}$ under $\mathrm{N}_{2}$. The mixture of standards was prepared by mixing and diluting the appropriate stock solutions to final concentrations between 0.5 and $400 \mathrm{pg} / \mu \mathrm{l}$. The internal standard $\left[{ }^{2} \mathrm{H}_{4}\right] 15-\mathrm{F}_{2 \mathrm{t}}$-IsoP was prepared in ethanol $(1 \mathrm{ng} / \mu \mathrm{l})$, and added to the mixture of standards and samples at a final concentration of $0.5 \mathrm{ng} / \mu \mathrm{l}$. Urine samples $(500 \mu \mathrm{l})$ were acidified with formic acid to $\mathrm{pH} 4 \cdot 5-5 \cdot 0$. Then, $10 \mu \mathrm{l}$ of $\beta$-glucuronidase $(90 \mathrm{U} / \mu \mathrm{l})$ were added to each sample and incubated at $37^{\circ} \mathrm{C}$ for $2 \mathrm{~h}$. Following incubation, the samples were cooled to room temperature, and then the $\mathrm{pH}$ was adjusted again to approximately $2.5 \mathrm{with}$ formic acid. After the addition of the isotope-labelled internal standard $\left[{ }^{2} \mathrm{H}_{4}\right] 15-\mathrm{F}_{2 \mathrm{t}}$-IsoP, the samples were subjected to solid-phase extraction on a C18 Sep-Pak cartridge (Waters Associates). The cartridges were preconditioned with $5 \mathrm{ml}$ methanol followed by $7 \mathrm{ml}$ of acid water (water acidified to $\mathrm{pH} 3$ with formic acid) before loading the samples. To remove interfering components, the cartridges were washed with $10 \mathrm{ml}$ acid water, $10 \mathrm{ml}$ acid water-methanol $(9: 1, \mathrm{v} / \mathrm{v})$, and $10 \mathrm{ml}$ heptane in succession. Then, the IsoP were eluted with $1 \mathrm{ml}$ methanol, the eluate was evaporated under $\mathrm{N}_{2}$, and the residues reconstituted with $100 \mu \mathrm{l}$ of the liquid chromatography starting mobile phase (A, see below). The IsoP were analysed by LC-ESI-QqQ-MS/MS using an Agilent 1100 HPLC series system; equipped with an autosampler with the temperature set to $8^{\circ} \mathrm{C}$; fitted with a Luna C18 column $(50 \times 2.0 \mathrm{~mm}$ inner diameter; $3.0 \mu \mathrm{m}$ packing; Phenomenex) and coupled to an AB Sciex API 3000 triple quadrupole mass spectrometer.

The instrument was operated in the negative-ion mode with a TurboIon spray source to obtain MS and MS/MS data, and the source temperature at $450^{\circ} \mathrm{C}$. In the separation step, elution was accomplished with a binary system consisting of $0.1 \%$ aqueous formic acid (A) and $0.1 \%$ formic acid in $\mathrm{CH}_{3} \mathrm{CN}(\mathrm{B})$, under an increasing linear gradient $(\mathrm{v} / \mathrm{v})$ of $\mathrm{B}$ $(t$ (min),$\% B): 0,10 ; 5,23 ; 12,50 ; 20,50 ; 21,100$ at a flow rate of $0.2 \mathrm{ml} / \mathrm{min}$. The IsoP were detected in multiple reaction monitoring experiments, and their identity was confirmed using the corresponding standard. Concentrations were interpolated in the calibration curves of each standard ( $R^{2}>0.99$ in all cases). Sample concentrations were calculated from the equation $y=m x+b$, as determined by weighted $\left(1 / x^{2}\right)$ linear regression of the standard curve.

The limit of detection was established at a signal:noise ratio of 3 , and the values were between 0.5 and $3.0 \mathrm{pg} / \mu \mathrm{l}$ depending on the standard. The limit of quantification was established at a signal:noise ratio of 10 , and the values were between 1.0 and $10.0 \mathrm{pg} / \mu \mathrm{l}$, depending on the standard. Peak integrations and signal:noise calculations were performed using 1.4.2 Analyst software (Applied Biosystems). The dwell time for multiple reaction monitoring experiments was $100 \mathrm{~ms}$, and the cycle time was $2 \mathrm{~s}$. The declustering potential and collision energy in multiple reaction monitoring mode were optimised for each standard in infusion experiments: $55 \mathrm{~V}$ and $28 \mathrm{eV}$ for $\left[{ }^{2} \mathrm{H}_{4}\right] 15-\mathrm{F}_{2 \mathrm{t}}$-IsoP $(357 \rightarrow 197) ; 55 \mathrm{~V}$ and $38 \mathrm{eV}$ for $15-\mathrm{F}_{2 \mathrm{t}}$-IsoP $(353 \rightarrow 193) ; 50 \mathrm{~V}$ and $22 \mathrm{eV}$ for 2,3 -dinor-15- $\mathrm{F}_{2 \mathrm{t}}$-IsoP $(325 \rightarrow 237) ; 55 \mathrm{~V}$ and $34 \mathrm{eV}$ for 
$5-\mathrm{F}_{2 \mathrm{t}}$-IsoP $\quad(353 \rightarrow 115) ; \quad 55 \mathrm{~V}$ and $33 \mathrm{eV}$ for $5-\mathrm{F}_{2 \mathrm{c}}$-IsoP $(353 \rightarrow 115) ; 55 \mathrm{~V}$ and $36 \mathrm{eV}$ for $8-\mathrm{F}_{2 \mathrm{t}}$-IsoP $(353 \rightarrow 127) ; 55 \mathrm{~V}$ and $33 \mathrm{eV}$ for $15-\mathrm{F}_{3 \mathrm{t}}$-IsoP $(351 \rightarrow 193)$.

The results are expressed as pg IsoP/mg creatinine to correct for urine concentration. Creatinine levels from the urine samples were determined by a colorimetric method using a commercial kit (C-cromatest Linear Chemicals) by measuring absorbance at $510 \mathrm{~nm}$ on a SpectraMax M5 spectrophotometer (Molecular Devices). Examples of the detection of IsoP in the standard mixture and in a sample are shown in Figs. S1 and S2 (available online).

\section{Statistical analyses}

Results from the fatty acid composition (see online supplementary Table S1) are expressed as means and standard deviations, and those from the measurements in rat samples are expressed as means with their standard errors. Levene's test and the Kolmogorov-Smirnov test were applied to assess variance equality and normal distribution, respectively. One-way ANOVA followed by Tukey's post hoc significance test was used when the assumptions of normality and equal variance were met. Otherwise, non-parametric tests (Kruskal-Wallis and Mann-Whitney $U$ rank-sum) were used to assess significance. Differences were considered significant at $P<0 \cdot 05$, and considered to indicate a tendency at $P<0 \cdot 1$. All statistical analyses were performed using the statistical package SPSS IBM version 19 for Windows.

\section{Results}

\section{Physical measurements}

Supplementation with EPA/DHA in different proportions did not modify either the animal body-weight time course, or the percentage of abdominal fat compared with the control group (data not shown).

\section{Measurements in plasma}

Plasma lipid profile. EPA/DHA significantly $(P<0 \cdot 05)$ lowered LDL-C at all the proportions tested, compared with that of the soyabean oil-supplemented control group (Table 1). The 2:1 and 1:2 EPA/DHA groups also decreased plasma total cholesterol concentration $(P<0 \cdot 05)$, while the 1:1 group had a non-significant lowering effect $(P<0 \cdot 1)$. TAG concentrations were significantly $(P<0 \cdot 05)$ decreased in the $1: 1$ and $2: 1$ groups. LDL-ox in plasma was significantly $(P<0.05)$ higher in all the EPA/DHA-supplemented SHROB rats than in the control group. No significant differences were found for HDL-cholesterol.

Glycaemia. Plasma glucose levels were not affected by EPA/DHA supplementation, while insulin levels were significantly higher in the $1: 1$ and $2: 1$ groups $(P<0 \cdot 05)$. The glycated $\mathrm{Hb}$ level was significantly lower in all the animals supplemented with EPA/DHA than in the control group (Table 1).

Inflammation. The levels of plasma CRP were significantly decreased by EPA/DHA supplementation at the 1:1 and 2:1 ratios $(P<0 \cdot 05$; Table 1$)$.

Endothelial dysfunction. EPA/DHA supplementation at the ratio of 1:2 increased vascular cell adhesion molecule-1 concentration in plasma $(P<0.001)$, but did not modify intercellular adhesion molecule-1 levels (Table 1).

Thrombotic activity. None of the EPA/DHA supplementation ratios had any influence on the plasma levels of plasminogen activator inhibitor-1 (Table 1).

\section{Endogenous antioxidant systems}

SOD and CAT activities. The activities of these antioxidant enzymes were evaluated in erythrocytes, kidneys, abdominal

Table 1. CVD risk factors in spontaneously hypertensive obese rats supplemented with EPA/DHA at different proportions (1:1, 2:1 and 1:2) and soyabean oil as the control diet

(Mean values with their standard errors)

\begin{tabular}{|c|c|c|c|c|c|c|c|c|c|}
\hline \multirow[b]{2}{*}{ Category } & \multirow[b]{2}{*}{ Parameter } & \multicolumn{2}{|c|}{ Control } & \multicolumn{2}{|c|}{$1: 1$} & \multicolumn{2}{|c|}{$2: 1$} & \multicolumn{2}{|c|}{$1: 2$} \\
\hline & & Mean & SEM & Mean & SEM & Mean & SEM & Mean & SEM \\
\hline \multirow[t]{5}{*}{ Plasma lipid profile } & Total cholesterol $(\mathrm{mg} / \mathrm{ml}) \S$ & $3 \cdot 2$ & 0.5 & $2 \cdot 1 \dagger$ & 0.2 & $1 \cdot 7^{\star \star}$ & 0.1 & $1.9^{*}$ & 0.2 \\
\hline & LDL-C (mg/ml)§ & 0.9 & 0.3 & $0.3^{*}$ & 0.1 & $0.2^{\star *}$ & 0.04 & $0.3^{*}$ & 0.1 \\
\hline & LDL-ox (ng/ml)\| & $154 \cdot 6$ & $23 \cdot 4$ & $279 \cdot 3^{\star \star \star}$ & $14 \cdot 6$ & $343 \cdot 4^{\star \star \star}$ & $18 \cdot 4$ & $292 \cdot 1^{\star \star}$ & $30 \cdot 6$ \\
\hline & HDL-C (mg/ml)\| & 0.8 & 0.1 & 0.7 & 0.1 & 0.6 & 0.03 & 0.7 & 0.1 \\
\hline & Total TAG $(\mathrm{mg} / \mathrm{ml})$ & $14 \cdot 6$ & 3.4 & $5 \cdot 7^{\star}$ & 0.8 & $5 \cdot 9^{*}$ & 0.6 & 9.9 & 0.2 \\
\hline \multirow[t]{3}{*}{ Glycaemia } & Glucose $(\mathrm{mg} / \mathrm{ml}) \|$ & 0.6 & 0.02 & 0.6 & 0.01 & 0.6 & 0.01 & 0.6 & 0.01 \\
\hline & Insulin $(\mathrm{ng} / \mathrm{ml}) \|$ & $5 \cdot 8$ & 1.0 & $12 \cdot 2^{\star *}$ & $2 \cdot 0$ & $9.8^{*}$ & 0.9 & $6 \cdot 8 \ddagger$ & 0.8 \\
\hline & Glycated Hb (\%) & $6 \cdot 8$ & 0.4 & $4 \cdot 2^{\star \star \star}$ & 0.1 & $4.5^{\star \star}$ & $0 \cdot 1$ & $5 \cdot 0^{\star \star}$ & 0.7 \\
\hline Inflammation & $\operatorname{CRP}(\mu \mathrm{g} / \mathrm{ml}) \|$ & 285.9 & $23 \cdot 8$ & $206 \cdot 0^{*}$ & $15 \cdot 9$ & $205 \cdot 2^{*}$ & $16 \cdot 0$ & $249 \cdot 6$ & 19.5 \\
\hline \multirow[t]{2}{*}{ Endothelial dysfunction } & VCAM $-1(\mu \mathrm{g} / \mathrm{ml}) \S$ & $2 \cdot 7$ & 0.3 & $3 \cdot 8$ & 0.1 & $3 \cdot 6$ & 0.4 & $5 \cdot 6^{\star \star \star} \ddagger$ & 0.7 \\
\hline & ICAM-1 (ng/ml)|| & 1.0 & $0 \cdot 1$ & 0.9 & $0 \cdot 1$ & $1 \cdot 1$ & $0 \cdot 1$ & 0.9 & 0.1 \\
\hline Thrombotic activity & PAI-1 $(\mu \mathrm{g} / \mathrm{ml}) \|$ & 11.6 & 0.6 & 10.5 & 0.8 & $10 \cdot 9$ & 0.5 & $12 \cdot 5$ & 1.2 \\
\hline
\end{tabular}

LDL-C, LDL-cholesterol; LDL-ox, oxidised LDL; HDL-C, HDL-cholesterol; CRP, C-reactive protein; VCAM-1, vascular cell adhesion molecule-1; ICAM-1, intercellular adhesion molecule-1; PAl-1, plasminogen activator inhibitor-1.

Mean value was significantly different from that of the control group: ${ }^{\star} P<0.05 ;{ }^{\star \star} P<0.01 ;{ }^{* \star} P<0.001$.

$\dagger$ Mean value tended to be different from that of the control group $(P<0.1)$.

$\ddagger$ Mean value was significantly different from that of the 1:1 group $(P<0.05)$.

$\S$ Comparisons were made using one-way ANOVA and Tukey's post hoc significance tests.

\| Comparisons were performed using Kruskal-Wallis and Mann-Whitney $U$ tests. 
fat, heart, brain and liver of the EPA/DHA-supplemented SHROB rats after 14 weeks of the experiment (Figs. 1 and 2). No differences were found in any of these enzymes in the liver.
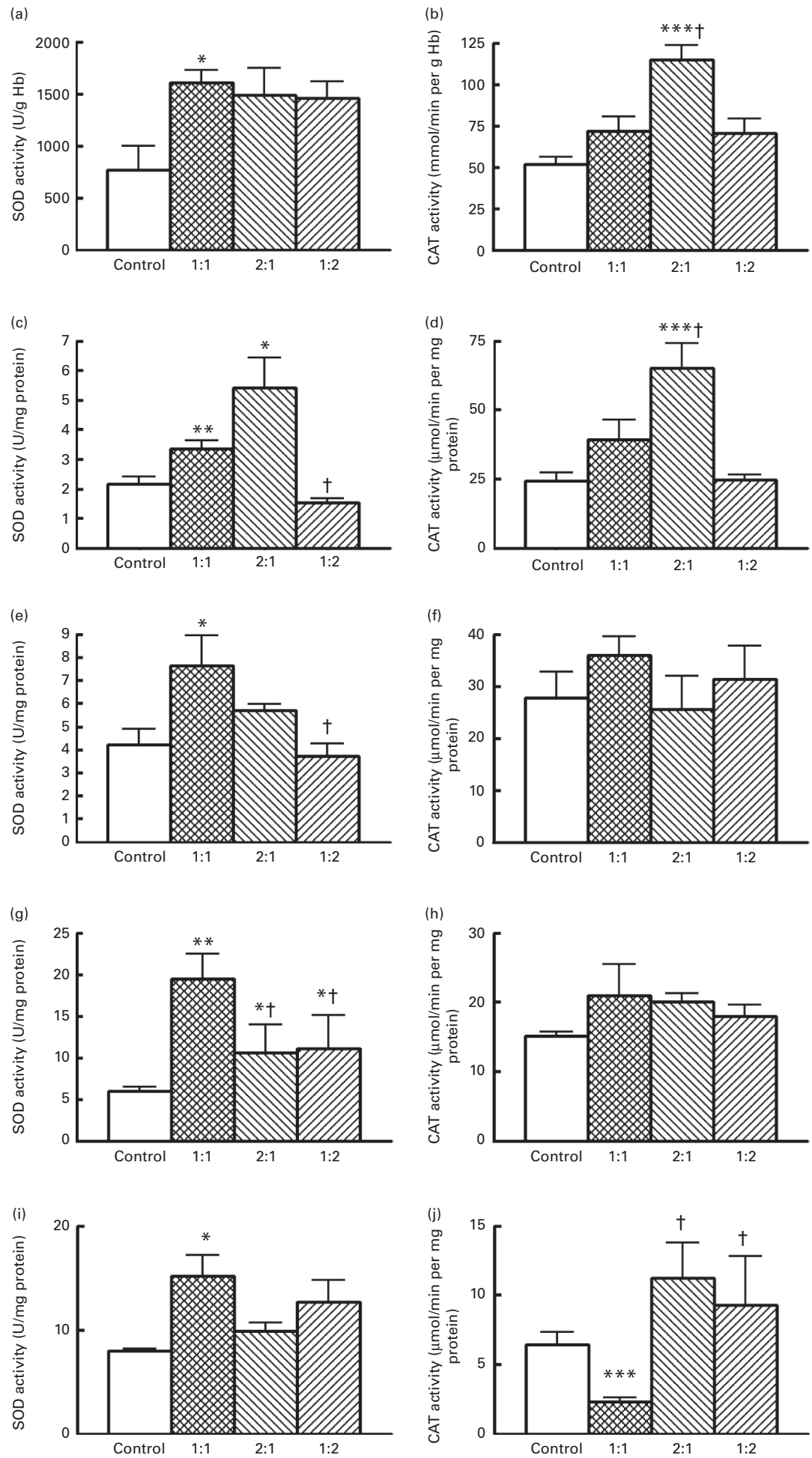

Fig. 1. ((a), (c), (e), (g), (i)) Superoxide dismutase and ((b), (d), (f), (h), (j)) catalase activities in different organs and tissues (((a), (b)) erythrocytes; ((c), (d)) kidneys; ((e), (f)) abdominal fat; ((g), (h)) heart; ((i), (j)) brain) of spontaneously hypertensive obese rats supplemented with EPA/DHA at different proportions $(1: 1,2: 1$ and 1:2) and soyabean oil as the control diet. Values are means, with their standard errors represented by vertical bars. Mean value was significantly different from that of the control group: ${ }^{*} P<0.05,{ }^{* \star} P<0.01,{ }^{* * *} P<0.001$ (Kruskal-Wallis and Mann-Whitney $U$ tests). $†$ Mean value was significantly different from that of the 1:1 EPA/DHA group $(P<0.05$; Kruskal-Wallis and Mann-Whitney $U$ tests). 

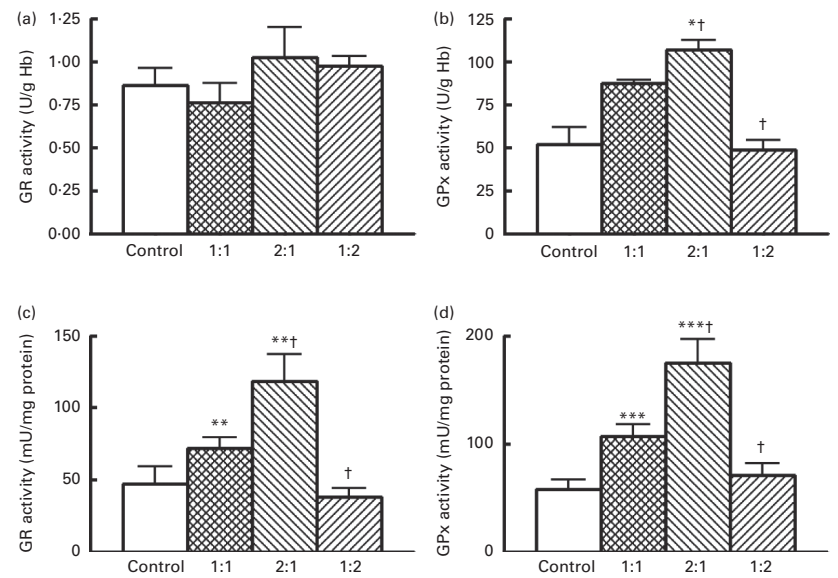

Fig. 2. ((a), (c)) Glutathione reductase and ((b), (d)) glutathione peroxidase activities in different organs and tissues ((a), (b) erythrocytes and (c), (d) kidney) of spontaneously hypertensive obese rats supplemented with EPA/DHA at different proportions (1:1, 2:1 and 1:2) and soyabean oil as the control diet. Values are means with their standard errors represented by vertical bars. Mean value was significantly different from that of the control group: ${ }^{\star \star} P<0.01,{ }^{\star \star *} P<0.001$ (Kruskal-Wallis and Mann-Whitney $U$ tests). † Mean value was significantly different from that of the 1:1 EPA/DHA group $(P<0.05$; Kruskal-Wallis and Mann-Whitney $U$ tests).

2:1 group $(P<0.05)$, and in the heart of the 1:2 EPA/DHA group $(P<0.05$; Fig. 1$)$.

CAT activity was significantly higher $(P<0 \cdot 001)$ in the erythrocytes and kidneys of the 2:1 EPA/DHA group, and lower in the brain of the 1:1 EPA/DHA group $(P<0 \cdot 001)$ (Fig. 1).

Glutathione system. The activity of the glutathioneassociated enzymes GR and GPx was measured in erythrocytes and kidneys, the compartments where SOD and CAT were most affected in comparison with the control group (Fig. 2). In erythrocytes, only GPx activity was detected $(P<0.01)$ in the $1: 1$ and 2:1 EPA/DHA groups, and in the kidneys, both enzymes were significantly activated $(P<0 \cdot 01)$ in the 1:1 and 2:1 EPA/DHA groups (Fig. 2). The ratio of the reduced:oxidised form of glutathione (GSH:GSSG) was lower $(P<0.05)$ in the kidneys of the 1:1 and 2:1 EPA/DHA groups (30.94 (SEM 3.09) and 36.73 (SEM 3.77)), respectively) than that found in the kidneys of both the control group (70.96 (SEM 18.02)) and the 1:2 EPA/DHA group $(76.70$ (SEM 7.58)).

\section{Urine isoprostanes}

Total urine IsoP (including both free and glucuronidated forms) were determined by LC-ESI-QqQ-MS/MS (Fig. 3). Also, 15- $\mathrm{F}_{3 \mathrm{t}}$-IsoP, directly derived from supplemented EPA/ DHA, was not detected in any of the samples, while $15-\mathrm{F}_{2 \mathrm{t}^{-}}$ IsoP, 5- $\mathrm{F}_{2 \mathrm{t}}$-IsoP, 5- $\mathrm{F}_{2 \mathrm{c}}$-IsoP and $8-\mathrm{F}_{2 \mathrm{t}}$-IsoP, endogenously derived from arachidonic acid, and 2,3-dinor-15- $\mathrm{F}_{2 \mathrm{t}}$-IsoP (a metabolite derived from $15-\mathrm{F}_{2 \mathrm{t}}$-IsoP) were detected in all the samples. Compared with the control group, the 1:2 EPA/ DHA group showed a tendency $(P<0 \cdot 1)$ towards a decrease in the levels of all the IsoP tested, while the 2:1 EPA/DHA group showed a tendency towards significantly lower levels of 2,3-dinor-15- $\mathrm{F}_{2 \mathrm{t}}$-IsoP $(P<0 \cdot 1)$.

\section{Discussion}

The present study focused on aspects of the action of $n-3$ PUFA in SHROB rats that were not addressed before, specifically their role in modulating OS and the influence of the relative proportions of EPA and DHA on both OS and significant markers of the MetS. The results of the present study indicate that EPA/DHA mixtures at different proportions clearly improve the altered lipid profile in SHROB rats; the three proportions tested $(1: 1,2: 1$ and 1:2) decreased LDL-C concentration and the proportions $1: 1$ and 2:1 decreased plasma TAG concentration. It has been reported that the effects of $n$ - 3 PUFA on the lipid profile were derived from their capacity to up-regulate the expression of genes encoding proteins involved in fatty acid oxidation, while simultaneously down-regulating genes encoding proteins involved in lipid synthesis $^{(25)}$. According to our data, mixtures of EPA and DHA that include more or equal amounts of shorter-chain EPA may be a better option than an excess of DHA for the control of dyslipidaemia in the MetS. A meta-analysis of clinical studies has revealed that both PUFA were similarly effective in decreasing TAG concentrations ${ }^{(26)}$; however, the results of the SHROB model appeared to differ from the outcome of human studies.

Chronic inflammation is closely linked to obesity ${ }^{(27)}$, and it is another relevant risk factor for $\mathrm{CVD}^{(28)}$. In the present study, we found a significant improvement in plasma CRP concentration in obese SHROB rats fed EPA/DHA, indicative of an amelioration of systemic inflammation. It has been suggested that $n-3$ PUFA show anti-inflammatory effects because they cause a partial displacement of the proinflammatory $n$-6 PUFA pathway and simultaneously generate anti-inflammatory resolvins (from EPA and DHA) and protectins (from DHA) ${ }^{(29,30)}$. Also, recent results have shown that $n-3$ PUFA inhibit NLRP3 inflammasome activation ${ }^{(31)}$. Again, in the results of the present study, those mixtures with more or equal amounts of EPA appear to be more effective than the DHA-rich 1:2 mixture. Indeed, the 1:2 mixture increased the concentration of vascular cell adhesion molecule-1, a marker of further inflammation, as previously reported in healthy WKY rats ${ }^{(9)}$. None of the mixtures had any effect on either intercellular adhesion molecule-1 or on the marker of thrombotic activity plasminogen activator inhibitor-1. The effects of $n$-3 PUFA on adhesion molecules appear to be controversial $^{(32)}$.

Regarding glucose metabolism, none of the EPA/DHA mixtures modified postprandial glycaemia, but all of them significantly decreased plasma glycated Hb levels. This is a beneficial effect of $n$-3 PUFA that has also been observed in normal WKY rats $^{(9)}$. Meanwhile, we observed that only the mixture with a higher proportion of EPA tended to increase insulin secretion. This seems to agree with a previous study in which DHA, but not EPA, prevented the increase in circulating insulin, induced by conjugated linoleic $\operatorname{acid}^{(33)}$. In contrast, other authors have reported that PUFA do not affect insulin secretion in healthy pigs ${ }^{(34)}$. Whereas the overall effect of PUFA on glucose metabolism is still controversial, there is evidence that EPA and DHA may act at different sites and involve different 

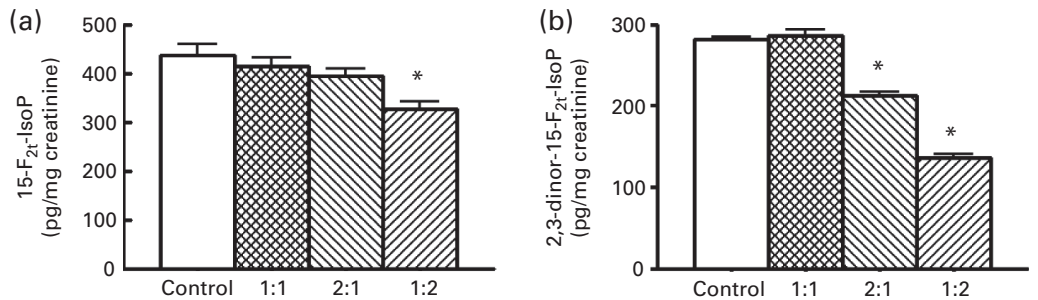

(c)

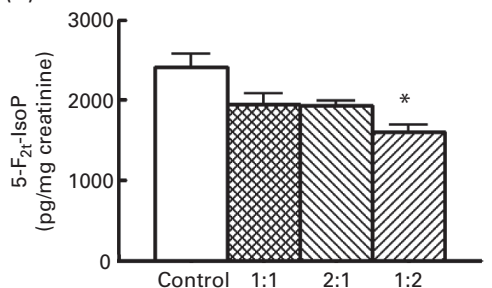

$(d)$

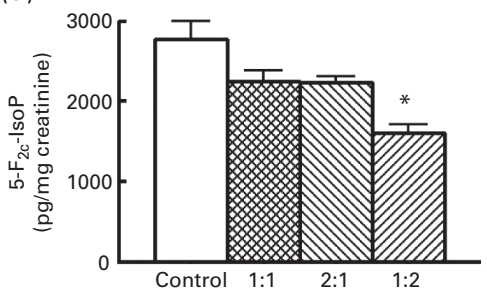

(e)

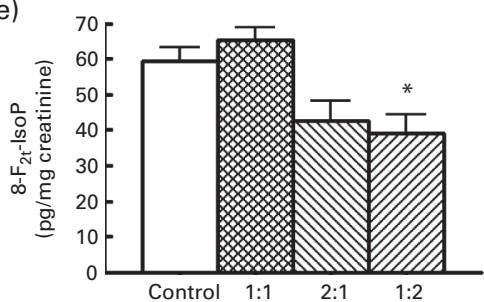<smiles>CCCCCC(O)/C=C/[C@H]1C(O)CC(O)[C@@H]1C/C=C/CCCC(=O)O</smiles>

$15-\mathrm{F}_{2 \mathrm{t}}$-IsoP<smiles>CCCCC/C=C/CC1C(O)CC(O)C1/C=C/C(O)CCCC(=O)O</smiles>

$5-\mathrm{F}_{2 \mathrm{c}}-\mathrm{IsoP}$<smiles>CCCCCC(O)/C=C/[C@H]1C(O)CC(O)[C@@H]1C/C=C/CC(=O)O</smiles>

2,3-dinor-15- $\mathrm{F}_{2 \mathrm{t}}$-IsoP<smiles>CCCCC/C=C/C[C@H]1[C@H](O)CC(O)[C@@H]1/C=C/C(O)CCCC(=O)O</smiles><smiles>CC/C=C\CC(O)/C=C/[C@H]1C(O)CC(O)[C@@H]1C/C=C/CCCC(=O)O</smiles>

$15-\mathrm{F}_{3 \mathrm{t}}-\mathrm{IsoP}$

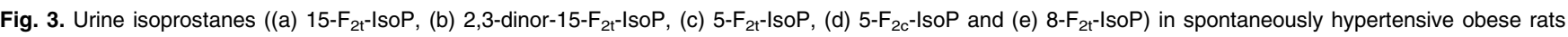
supplemented with EPA/DHA at different proportions (1:1, 2:1 and 1:2) and soyabean oil as the control diet. Values are means, with their standard errors represented by vertical bars. *Mean value tended to be different from that of the control group $(P<0 \cdot 1$; Kruskal-Wallis and Mann-Whitney $U$ tests). The structures of the respective compounds are also shown. $15-\mathrm{F}_{3 \mathrm{t}}$-IsoP was not detected in any of the samples.

mechanisms related to insulin resistance ${ }^{(35)}$. Consequently, mixtures at different proportions yield different results.

As OS is another factor that has been closely associated with the $\mathrm{MetS}^{(3)}$, we examined the effect of EPA/DHA supplementation on endogenous antioxidant systems and IsoP as the 'gold' standard marker of $\mathrm{OS}^{(36,37)}$. We found that EPA/ DHA at the ratio of 1:1 increases SOD activity in the adipose tissue, erythrocytes, heart and brain of SHROB rats.
In a previous study, we described that fat and erythrocytes of SHROB rats exhibited decreased SOD activity compared with WKY rats ${ }^{(14)}$. Herein, we show that EPA/DHA supplementation at the ratio of 1:1 can increase SOD activities to values close to those found in healthy WKY rats. We also previously reported that antioxidant enzyme activities in the heart and brain of SHROB and normal WKY rats were similar $^{(14)}$. In these organs, $n-3$ PUFA appear to activate this 
protective system in SHROB rats. This activation has also been observed in the erythrocytes of healthy WKY rats supplemented with EPA/DHA at the ratio of $1: 1^{(9)}$. Moreover, EPA/DHA mixtures also increase the activity of other enzymes, such as CAT and GPX in erythrocytes, with this effect being more marked in rats supplemented with EPA/DHA at the ratio of $2: 1$. In the kidneys, an organ particularly affected in the present rat model ${ }^{(12)}$, EPA/DHA supplementation at the ratio of $2: 1$ was more effective than that at $1: 1$.

We have also previously reported that endogenous antioxidant systems in the kidneys were not altered in SHROB rats compared with WKY rats ${ }^{(14)}$. However, in the present study, EPA/DHA at the ratio of 2:1 caused an increase in the activity of different antioxidant enzymes (SOD, CAT, GR and GPx), and a displacement of the glutathione system towards accumulation of GSSG (a decreased GSH:GSSG ratio). All these results suggest that $\mathrm{EPA} / \mathrm{DHA}$ supplementation triggers some OS with subsequent activation of defence mechanisms. The changes in the markers of OS (plasma LDL-Ox and urine IsoP) examined in the present study are consistent with these aforementioned observations.

EPA/DHA mixtures at all the three ratios significantly increased plasma LDL-Ox in SHROB rats. A similar increase in LDL-ox after supplementation with $n$-3 PUFA has previously been described in an animal model of the MetS ${ }^{(38)}$. These authors hypothesised that because $n$ - 3 PUFA are incorporated into cell membranes, they cause an increase in the peroxidability index, i.e. the number of sites susceptible to lipid peroxidation. However, at this point, it should be noted that the PUFA supplemented in the present study did not directly contribute to the circulating lipid peroxides, as demonstrated by the absence of $15-\mathrm{F}_{3 \mathrm{t}}$-IsoP, which would have been derived from EPA and DHA. In the apparent contradiction of the results for plasma LDL-ox, the supplemented rats presented lower levels of urine IsoP (Fig. 3), particularly the 1:2 EPA/DHA group. This means that the overall effect of EPA and DHA in the organism, as evidenced by the levels of endogenous lipid oxidation, appears to be antioxidant. This overall antioxidant effect may be caused by the activation of defence systems, which would also agree with our previous observation that plasma antioxidant capacity increases in WKY rats after supplementation with $n-3 \operatorname{PUFA}^{(9)}$.

Taken together, it seems that the results of the present study indicate that EPA is especially relevant to the activation of endogenous antioxidant systems and the decrease in circulating TAG and CRP levels, while DHA is more effective in lowering IsoP. These differential effects may be due to the different effects of EPA and DHA on signalling pathways, or on the expression of certain genes, particularly on those related to the activation of antioxidant enzymes. It has been reported that EPA and DHA differently influence transduction pathways related to cell growth, thereby affecting different biological processes ${ }^{(39)}$, the release of inflammatory cytokines by peripheral blood mononuclear cells ${ }^{(40)}$, and the expression of genes involved in lipid metabolism ${ }^{(41)}$.

Also, the incorporation of either EPA or DHA into cell membranes has different effects on their structure, as shown for endothelial cells, lymphocytes and in ion channel effects, as observed in several cellular models ${ }^{(32)}$. Another relevant factor is that EPA and DHA may exhibit specific interactions with other food components such as vitamin $\mathrm{E}$ that influence oxidative status, and act together with DHA in the expression of certain genes ${ }^{(42)}$. Certainly, our observations indicate that the proportions of EPA and DHA should be carefully considered when carrying out studies with $n$-3 PUFA or when comparing results from different studies.

In short, supplementation of SHROB rats, a model of the MetS, with EPA/DHA mixtures at different proportions significantly lowered plasma total cholesterol and LDL-C concentrations, compared with the control group, given a standard diet. Specific mixtures significantly decreased inflammation (CRP levels, 1:1 and 2:1 ratios), increased the activity of antioxidant enzymes (SOD in erythrocytes and abdominal fat, 1:1 ratio; SOD, CAT, GR, GPx in kidneys, 1:2 ratio), and showed a tendency towards decreasing excretion of urinary IsoP (mainly 1:2 ratio). More LDL-ox was observed in all the supplemented groups. These results are compatible with the stimulation of antioxidant defences by EPA/DHA mixtures, leading to lower OS, together with a reduction in CVD risk factors such as LDL-C and inflammation in SHROB rats.

\section{Supplementary material}

To view supplementary material for this article, please visit http://dx.doi.org/10.1017/S0007114514004437

\section{Acknowledgements}

The authors thank Lorena Barros and $\mathrm{M}^{\mathrm{a}}$ Jesus Gonzalez for technical support, and Christopher Evans for language revision. The authors also thank AFAMSA (Vigo, Spain) for the provision of the commercial fish oil AFAMPES 121 EPA.

The present study work was supported by the Spanish Ministry of Science and Innovation and of Economy and Competitiveness (grant no. AGL2009-12374-C03-01, -02 and -03; AGL2013-49079-C2-1,2-R, respectively). The authors acknowledge the Panamanian Government (SENACYT/IFARHU) and the Spanish Ministry of Science and Innovation for their predoctoral fellowships to E. M.-T. and L. M., respectively. They also acknowledge ISCIII for the postdoctoral contract 'Sara Borrell' to J. P.-J. (CD09/00068). None of the funders had any role in the design, analysis and findings of the study or in the writing of this article.

The contributions of the authors are as follows: I. M. and J. L. T. designed the research; E. M.-T., J. P.-J., M. R., M. G., N. T., M. M.-C., O. J. and L. M. carried out the experimental work; E. M.-T., J. P.-J., S. R.-R. and J. L. T. analysed the data; E. M.-T., J. P.-J. and S. R.-R. wrote the first draft of the manuscript; J. P.-J. and J. L. T. had primary responsibility for the final content. All the authors read and approved the final version of the manuscript.

The authors declare there are no conflicts of interests. 


\section{References}

1. Eckel RH, Alberti KGMM, Grundy SM, et al. (2010) The metabolic syndrome. Lancet 375, 181-183.

2. Festa A, D'Agostino R, Howard G, et al. (2000) Chronic subclinical inflammation as part of the insulin resistance syndrome: the Insulin Resistance Atherosclerosis Study (IRAS). Circulation 402, 42-47.

3. Armutcu F, Ataymen M, Atmaca H, et al. (2008) Oxidative stress markers, C-reactive proteins and heat shock protein 70 levels in subjects with metabolic syndrome. Clin Chem Lab Med 46, 785-790.

4. Aguilera AA, Díaz GH, Barcelata ML, et al. (2004) Effects of fish oil on hypertension, plasma lipids and tumor necrosis factor- $\alpha$ in rats with sucrose-induced metabolic syndrome. J Nutr Biochem 15, 350-357.

5. Huang YJ, Fang VS, Juan CC, et al. (1997) Amelioration of insulin resistance and hypertension in a fructose-fed rat model with fish oil supplementation. Metabolism 46, $1252-1258$.

6. Poudyal H, Panchal SK, Diwan V, et al. (2011) Omega-3 fatty acids and metabolic syndrome: effects and emerging mechanisms of action. Prog Lipids Res 50, 372-387.

7. Lorente-Cebrián AS, Costa AGV, Navas-Carretero S, et al. (2013) Role of omega-3 fatty acids in obesity, metabolic syndrome, and cardiovascular diseases: a review of the evidence. J Physiol Biochem 69, 633-651.

8. Burghardt PR, Kemmerer ES, Buck BJ, et al. (2010) Dietary n-3:n-6 fatty acids ratio differentially influence hormonal signature in a rodent model of metabolic syndrome relative to healthy controls. Nutr Metab 7, 53.

9. Lluís L, Taltavull N, Muñoz-Cortés M, et al. (2013) Protective effect of the omega-3 polyunsaturated fatty acids: eicosapentaenoic acid/docosahexaenoic acid 1:1 ratio on cardiovascular disease risk markers in rats. Lipids Health Dis 12, 140.

10. Méndez L, Pazos M, Gallardo JM, et al. (2013) Reduced protein oxidation in Wistar rats supplemented with marine omega-3 PUFA. Free Radical Biol Med 55, 8-20.

11. Takaya K, Ogawa Y, Hiraoka J, et al. (1996) Nonsense mutation of leptin receptor in the obese spontaneously hypertensive Koletsky rat. Nat Genet 14, 130-131.

12. Ernsberger P, Koletsky RJ \& Friedman JE (1999) Molecular pathology in the obese spontaneous hypertensive Koletsky rats: a model of syndrome X. Ann NY Acad Sci 892, 315-318.

13. Aleixandre de Artiñano A \& Miguel Castro M (2009) Experimental rat models to study the metabolic syndrome. Br J Nutr 102, 1246-1253.

14. Molinar-Toribio E, Pérez-Jiménez J, Ramos-Romero S, et al. (2014) Cardiovascular-disease related parameters and oxidative stress in SHROB rats, a model for metabolic syndrome. PLOS ONE 9, e104637.

15. García-Hernández VM, Gallar M, Sánchez-Soriano J, et al. (2013) Effect of omega-3 dietary supplements with different oxidation levels in the lipidic profile of women: a randomized controlled trial. Int J Food Sci Nutr 64, 993-1000.

16. Cohen G, Dembiec D, Marcus J, et al. (1970) Measurement of catalase activity in tissue extracts. Anal Biochem 1970, $30-38$.

17. Misra HP \& Fridovich I (1972) The role of superoxide anion in the autoxidation of epinephrine and a simple assay for superoxide dismutase. J Biol Chem 247, 3170-3175.

18. Goldberg DM \& Spooner RJ (1983) Glutathione reductase. In Methods of Enzymatic Analysis, 3rd ed., pp. 258-265 [HU Bergmeyer, editor]. Weinheim, Germany: Verlag Chemie.
19. Wheeler CR, Salzman JA, Elsayed NM, et al. (1990) Automated assays for superoxide dismutase, catalase, glutathione peroxidase, and glutathione reductase activity. Anal Biochem 184, 193-199.

20. Hissin PJ \& Hilf RA (1976) Fluorometric method for determination of oxidized and reduced glutathione in tissues. Anal Biochem 74, 214-226.

21. Drabkin D \& Austin J (1935) Spectrophotometric studies. II. Preparations from washed blood cells; nitric oxide hemoglobin and sulfhemoglobin. $J$ Biol Chem 112, 51-65.

22. Bradford MM (1976) A rapid and sensitive method for the quantitation of microgram quantities of protein utilizing the principle of protein-dye binding. Anal Biochem $\mathbf{7 2}$, $248-254$.

23. Langhorst ML, Hastings MJ, Yokoyama WH, et al. (2010) Determination of $\mathrm{F}_{2}$-isoprostanes in urine by online solid phase extraction coupled to liquid chromatography with tandem mass spectrometry. J Agric Food Chem 58, 6614-6620.

24. Masoodi M \& Nicolaou A (2006) Lipidomic analysis of twenty-seven prostanoids and isoprostanes by liquid chromatography/electrospray tandem mass spectrometry. Rapid Commun Mass Spectrom 20, 3023-3029.

25. Lombardo YB \& Chicco AG (2006) Effects of dietary polyunsaturated dietary $n$ - 3 fatty acids on dyslipidaemia and insulin resistance in rodents and humans. A review. J Nutr Biochem 17, $1-13$.

26. Wei MY \& Jacobson TA (2011) Effects of eicosapentaenoic acid versus docosahexaenoic acid on serum lipids: a systematic review and meta-analysis. Curr Atheroscler Rep 13, 474-483.

27. Yudkin JS, Steuhower CDA, et al. (1999) C-reactive protein in healthy subjects: associations with obesity, insulin resistance, and endothelial dysfunction: a potential role for cytokines originating from adipose tissue? Arterioscl Throm Vas 19, 972-978.

28. Willerson JT \& Ridker PM (2004) Inflammation as a cardiovascular risk factor. Circulation 109, 2-10.

29. Serhan CN, Hong S, Gronert K, et al. (2002) Resolvins: a family of bioactive products of omega- 3 fatty acid transformation circuits initiated by aspirin treatment that counter proinflammation signals. J Exp Med 196, 1025-1037.

30. Serhan CN, Gotlinger K, Hong S, et al. (2006) Antiinflammatory actions of neuroprotectin D1/protectin D1 and its natural stereoisomers: assignments of dihydroxycontaining docosatrienes. J Inmunol 176, 1848-1859.

31. Yan Y, Jiang W, Spinetti T, et al. (2013) Omega-3 fatty acids prevent inflammation and metabolic disorder through inhibition of NLRP3 inflammasome activation. Immunity 38, $1154-1163$.

32. Mozaffrian D \& Wu JHY (2012) (n-3) Fatty acids and cardiovascular health: are effects of EPA and DHA shared or complementary? J Nutr 142, 614-625.

33. Vemuri M, Kelley DS, Mackey BD, et al. (2007) Docosahexaenoic acid (DHA) but not eicosapentaenoic acid (EPA) prevents trans-10, cis-12 conjugated linoleic acid (CLA)-induced insulin resistance in mice. Metab Syndr Relat Disord 5, 315-322.

34. Castellano CA, Audet I, Laforest JP, et al. (2010) Fish oil diets do not improve insulin sensitivity and secretion in healthy adult male pigs. Br J Nutr 103, 189-196.

35. Fedor D \& Kelley DS (2009) Prevention of insulin resistance by $n-3$ polyunsaturated fatty acids. Curr Opin Clin Nutr 12, $138-146$.

36. Jahn U, Galano JM \& Durand T (2008) Beyond prostaglandins-chemistry and biology of cyclic oxygenated metabolites 
formed by free-radical pathways from polyunsaturated fatty acids. Angew Chem Int Ed 47, 5894-5955.

37. Roberts LJ \& Morrow JD (2000) Measurement of F(2)isoprostanes as an index of oxidative stress in vivo. Free Radic Biol Med 28, 505-513.

38. Ferreira de Castro GS, Dos Santos RA, Portari GV, et al. (2012) Omega-3 improves glucose tolerance but increases lipid peroxidation and DNA damage in hepatocytes of fructose-fed rats. Appl Physiol Nutr Metab 37, 233-240.

39. Serini S, Fasano E, Piccioni E, et al. (2011) Differential anti-cancer effects of purified EPA and DHA and possible mechanisms involved. Curr Med Chem 18, 4065-4075.
40. Serrín E, Bizarro A, Piccioni E, et al. (2012) EPA and DHA differentially affect in vitro inflammatory cytokine release by peripheral blood mononuclear cells from Alzheimer's patients. Curr Alzheimer Res 9, 913-923.

41. Caputo M, Zirpoli H, Torino G, et al. (2011) Selective regulation of UGT1A1 and SREBP-1C mRNA expression by docosahexaenoic, eicosapentaenoic, and arachidonic acids. J Cell Physiol 226, 187-193.

42. Caputo M, Eletto D, Torino G, et al. (2008) Cooperation of docosahexaenoic acid and vitamin $\mathrm{E}$ in the regulation of UDP-glucuronosyltransferase mRNA expression. $J$ Cell Physiol 215, 765-770. 